\title{
Synthesis, characterization, magnetic properties of cobalt coordination polymer based on 4,4'-Oxybisbenzoic acid and 1,4-bis(pyrid-4-yl)benzene
}

\author{
Fengping Xiao, Peng $\mathrm{Hu}^{\mathrm{a},{ }^{*}}$ \\ Environmental and Chemical Engineering College, Zhaoqing University, Zhaoqing 526061, China. \\ ahp8286799@163.com
}

Keywords: Crystal structure, Cationic chain, Coordination polymer, Magnetic properties.

\begin{abstract}
A compound with cation chains and monomers, $\left[\mathrm{Co} 1 / 2(\text { bpbenz) })_{1 / 2}\right]^{+}[\mathrm{Co} 1 / 2(\mathrm{oba})]^{-}$(1) (bpbenz $=1$,4-bis(pyrid-4-yl)benzene and $\mathrm{H}_{2}$ oba $=4,4$-Oxybisbenzoic acid) has been synthesized under hydrothermal condition. Single crystal X-ray diffraction analyses reveal that in compound 1, the monomer $\left[\mathrm{Co}_{1 / 2}(\mathrm{oba})\right]^{-}$anions stack in three orientations to generate a $1 \mathrm{D}$ pseudo channels encapsulation of cationic chains $\left[\mathrm{Co}_{1 / 2}(\mathrm{bpbenz})_{1 / 2}\right]^{+}$. Magnetic studies indicate that compound $\mathbf{1}$ exhibit weak antiferromagnetic interactions.
\end{abstract}

\section{Introduction}

Coordination polymers have been widely studied in material science because of their potential applications in luminescence, magnetism, catalysis, drug delivery and molecule storage [1-5]. However, the achievements of these applications require careful design of structures. As is known, ligand and metal ion are two necessary components leading to extended supramolecular multi-metallic assemblies. Among all the ligands, exo-bidentate ligands such as 4, 4'-bipyridine or pyrazine are regarded as excellent ancillary ligands for constructing novel structures [6]. As the bidentate bridge with different conformations may leading to the formation of different motifs like 1D chain, 2D layer and 3D network [7]. Besides ligand, the choice of metal ions is also important as different metal have their own preferred properties. For example, nickel-based complexes have owned considerable interest as pre-catalysts, fluorescent emission and the identification of coordination environments in biological nickel centers [8]. Another transition metal of Co-based complexes is also identified with excellent magnetism properties.

Based on these research backgrounds, herein, we choose the exo-bidentate ligands 1,4-bis(pyrid-4-yl) benzene (bpbenz) as bridging ligands in combination with 4,4'-Oxybisbenzoic acid( $\mathrm{H}_{2} \mathrm{Oba}$ ) (Scheme 1) to build new coordination polymers. A one-dimensional polymer $\left[\mathrm{Co}_{1 / 2}(\mathrm{bpbenz})_{1 / 2}\right]^{+}\left[\mathrm{Co}_{1 / 2}(\mathrm{oba})\right]^{-}(\mathbf{1})$ has been constructed successfully. We report herein the synthesis, crystal structure, and magnetic properties of it. Magnetic data reveal weak antiferromagnetic coupling for compound $\mathbf{1}$. It's worth noting that complex $\mathbf{1}$ has been reported recently but its magnetic properties have never been studied before [9].
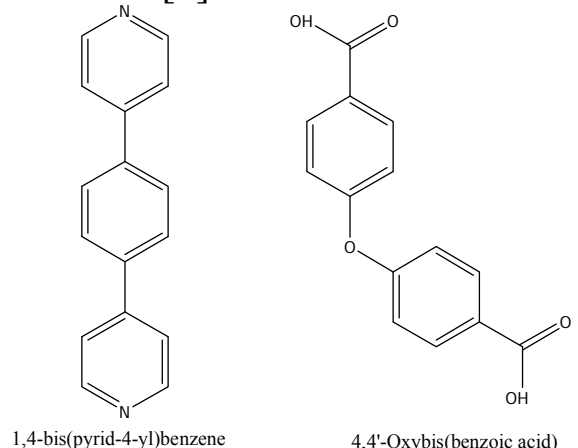

Scheme 1 Structural formulas of all ligands 


\section{Experimental}

\subsection{Synthesis of [Co1/2(bpbenz)1/2]+[Co1/2(oba)]- (1)}

The mixture of $\mathrm{CoCl}_{2} .6 \mathrm{H}_{2} \mathrm{O}((0.0476 \mathrm{~g}, 0.2 \mathrm{mmol})), 4,4$ '-Oxybisbenzoic acid $(0.0516 \mathrm{~g}, 0.2 \mathrm{mmol})$, 1,4-bis(pyrid-4-yl)benzene $(0.0465 \mathrm{~g}, 0.2 \mathrm{mmol})$ and $10 \mathrm{~mL}$ distilled water were sealed in a $25 \mathrm{~mL}$ Teflon-lined stainless steel vessel. The mixture was heated at $160^{\circ} \mathrm{C}$ for $70 \mathrm{~h}$ and cooled to room temperature at a rate of $10^{\circ} \mathrm{C} / \mathrm{h}$. Subsequently, red block-shaped crystals were obtained by filtration, washed with distilled water and dried in air. Anal. Yield $40.2 \%$ (based on $\mathrm{CoCl}_{2} .6 \mathrm{H}_{2} \mathrm{O}$ ). Calcd. for $\mathrm{C}_{22} \mathrm{H}_{22} \mathrm{CoNO}_{9}(\mathrm{Mr}=503.34)$ : C 52.50, H 4.41, N $2.76 \%$; found: C 52.91, H 4.23, N 2.60 \%. IR (KBr pellet, $\mathrm{cm}^{-1}$ ): 3239 s, 2928 s, 2378 s, 1663 m, 1604 s, 1543 s, 1365 s, 1227 s, 1157 m, 1007 m, 888 m, $799 \mathrm{~s}, 710 \mathrm{~m} .492 \mathrm{~m}$.

\subsection{X-ray Crystallography}

The single crystal structure data for compound 1 was collected on a Bruker SMART1000 CCD with graphite monochromatic $\mathrm{Mo} / \mathrm{K}$ a radiation $(\lambda=0.71073 \AA)$ at room temperature. The structure was solved by direct method with SHELXS-97 program and refined by full-matrix least-squares on $F^{2}$ with SHELXL-97 package. All non-hydrogen atoms were refined anisotropically, and hydrogen atoms were located and included at their calculated positions. CCDC No. 1524320

\section{Results and Discussion}

\subsection{X-ray diffraction of compound 1}

As shown in Fig. 1, compound $\mathbf{1}$ crystallizes in the $P-1$ space group, the asymmetric units of $\mathbf{1}$ are composed of one $\left[\mathrm{Co} 1 / 2(\text { bpbenz })_{1 / 2}\right]^{+}$cationic chain and one $[\mathrm{Co} 1 / 2(\mathrm{oba})]^{-}$anion. Both two $\mathrm{Co}^{2+}$ anions are six-coordinated in octahedral geometry. $\mathrm{Col}$ connect to four $\mathrm{O}$ atoms from water molecules in the basal plane (O8, $\mathrm{O} 9$ and $\mathrm{O} 8 \# 1, \mathrm{O} 9 \# 1)$ and two $\mathrm{N}$ atoms from two bpbenz ligands in the axial position $(\mathrm{N} 1, \mathrm{~N} 1 \# 1)$; the other $\mathrm{Co}^{2+}$ anion connect to four $\mathrm{O}$ atoms from water molecules in the basal plane $(\mathrm{O} 6, \mathrm{O} 7, \mathrm{O} 6 \# 2, \mathrm{O} 7 \# 2)$ and two $\mathrm{O}$ atoms from oba ${ }^{2-}$ ligands in the axial position $(\mathrm{O} 4$, O4\#2) The bond lengths of Co-O in the range 2.067 (1)- 2.124 (1) $\AA$ while bond lengths of Co1-N1 is 2.181(1) A. The bpbenz ligand connects two symmetry-related Co1(II) ions and each Co1(II) ion is connects to two different bpbenz ligands, thus generating a 1D chain (Fig.1).

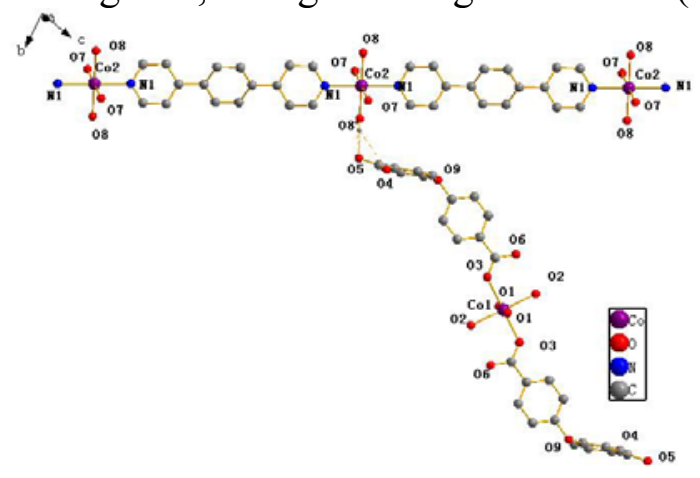

Fig. 1 Molecular structure of compound 1, all hydrogen atoms are omitted for clarity.

\subsection{Powder X-ray diffraction and Thermogravimetric analysis of compound 1}
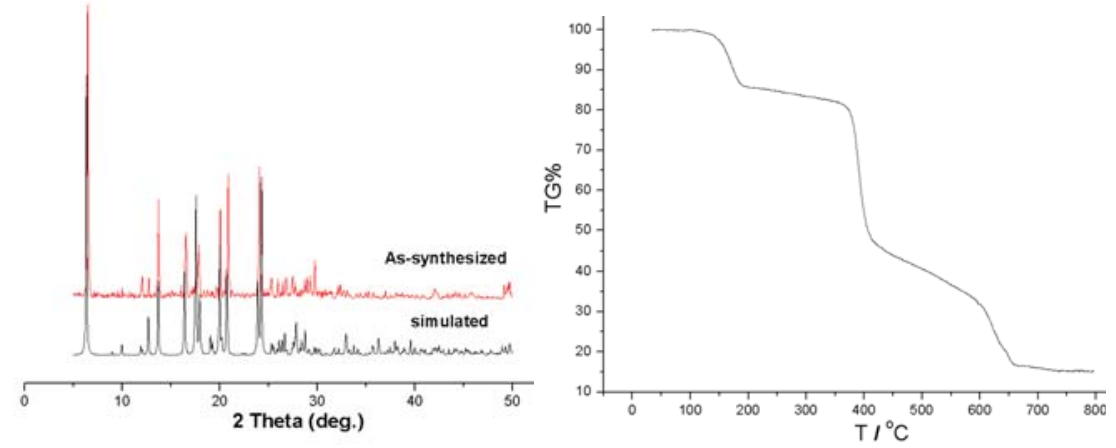

Fig. 2 PXRD patterns (left) and TGA trace (right) of compound 1 
To check the crystallinity and purity, compound 1 was measured by X-ray powder diffraction at room temperature (Fig.2 left). XRD results show that this compound is of high crystallinity with sharp Bragg reflections. The diffraction peaks agree well with simulated pattern.

In order to characterize the thermal stability of compound $\mathbf{1}$, we performed the thermogravimetric analysis (TGA). The relevant experiments for the crystalline samples of compound $\mathbf{1}$ was performed in a $\mathrm{N}_{2}$ atmosphere wherein the sample was heated from room temperature to $800{ }^{\circ} \mathrm{C}$ at a rate of $10^{\circ} \mathrm{C} / \mathrm{min}$. The TG curve of it is shown in Fig. 2 (right). The TG curve show a weight loss in the temperature range of $120-190{ }^{\circ} \mathrm{C}$ corresponds to the removal of four lattice water molecules (calcd. $14.3 \%$, obsd. $14.2 \%$ ). Upon further heating, a sharp weight loss was observed from $360{ }^{\circ} \mathrm{C}$ to $410^{\circ} \mathrm{C}$ corresponds to the removal of four oba ligands (calcd. 50.9\%, obsd. 51.3\%). Finally, compound 1 was completely degraded into $\mathrm{CoO}$ with total loss of $84.9 \mathrm{wt} \%$ (calcd. $85.1 \mathrm{wt} \%$ ).

\subsection{Magnetic properties of compound 1}

The temperature dependence of the molar magnetic susceptibilities of compound 1 was measured from 300 to $2.0 \mathrm{~K}$ in an applied field of $2 \mathrm{kOe}$. As shown in Fig. 3, the $\chi_{\mathrm{M}} T$ value at room temperature is $2.91 \mathrm{~cm}^{3} \cdot \mathrm{K} \cdot \mathrm{mol}^{-1}$, which is higher than expected for the uncoupling value for a high-spin $\mathrm{Co}$ (II) center $\left(\chi_{\mathrm{M}} T=1.87 \mathrm{~cm}^{3} \cdot \mathrm{K} \cdot \mathrm{mol}^{-1}\right)$ due to orbital angular momentum. Upon cooling, $\chi_{\mathrm{M}} T$ decreases continuously to reach $0.032 \mathrm{~cm}^{3} \cdot \mathrm{K} \cdot \mathrm{mol}^{-1}$ at $2.0 \mathrm{~K}$. The decreasing trend of $\chi \mathrm{M} T$ value with the temperature suggests the presence of antiferromagnetic interactions.

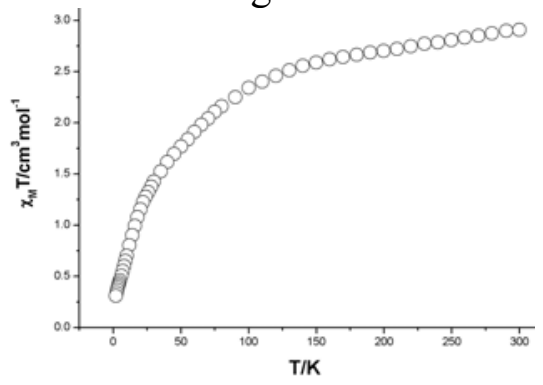

Fig. 3 Temperature dependence of the $\chi \mathrm{MT}$ product of compound 1.

\section{Summary}

In summary, A compound of Co based 1,4-bis(pyrid-4-yl)benzene and 4,4'-Oxybisbenzoic acid has been successfully synthesized and characterized. The crystal structural analyses indicate that monomer anions stack in three orientations to generate a 1D pseudo channels encapsulation of one-dimensional cationic chains. The magnetic studies indicate that compound $\mathbf{1}$ shows antiferromagnetic coupling between Co (II) ions.

\section{References}

[1]. J. Teufel, H. Oh, M. Hirscher, et al. MFU-4-A metal-organic framework for highly effective H2 /D2 separation. Adv. Mater.Vol. 25 (2013) p. 635-639.

[2]. R.T. Woodward, L.A. Stevens, R. Dawson, et al. Water-and acid-tolerant polymer sponges for chemoselective carbon dioxide cap-ture, J. Am. Chem. Soc. Vol.136 (2014) p. 9028-9035.

[3]. J. Yu, Y. Cui, Y. Yang, et al. Second-order nonlinear optical activity induced by ordered dipolar chromophores confined in the pores of an anionic metal-organic framework, Angew. Chem. Int. Ed. Vol.51 (2012) p. 10542-10545.

[4]. X. Bao, H.J. Shepherd, L. Salmon, et al. The effect of an active guest on the spin crossover phenomenon, Angew. Chem. Int. Ed. Vol.52 (2013) p. 1198-1202.

[5]. L. Qin, M.X. Zheng, Z.J. Guo, et al. One non-interpenetrated chiral porous multifunctional metal-organic framework and its applications for sensing small solvent molecules and adsorption, Chem. Commun. Vol.51 (2015) p. 2247-2249. 
[6]. L. Qin, Y.Z. Li, Z.J. Guo, et al. Structural diversity and properties of six 2D or 3D metal-organic frameworks based on thiophene-containing ligand, Cryst. Growth Des. Vol.12 (2012) p. 5783-5791.

[7]. B. Moulton, M.J. Zaworotko, From molecules to crystal Engineering: supra-molecular isomerism and polymorphism in network solids, Chem. Rev. Vol.101 (2001) p. 1629-1658.

[8]. S.Kongchoo, K.Chainok, A.Kantacha, et al. Syntheses, crystal structures, spectroscopy, and catalytic properties of two nickel-based hexaazamacrocyclic complexes with carboxylate ligands. Inorg. Chem. Commun. Vol. 83 (2017) p. 97-102.

[9]. L.Qin, P.Y. Chen, G.C.Zhao, et al. Encapsulation of one-dimensional cation chains into pseudo-channels by varying monomers with opposite charge. J. Mol. Struct. Vol. 1125 (2016) p. 546-549. 